\title{
What do we learn from the classical turning surface of the Kohn-Sham potential as electron number is varied continuously?
}

\author{
Tim Gould* \\ Qld Micro- and Nanotechnology Centre, Griffith University, Nathan, Qld 4111, Australia \\ Benjamin T. Liberles \\ Cooper City High School, Cooper City, FL 33328, USA \\ John P. Perdew \\ Department of Physics, Temple University, Philadelphia, PA 19122, USA and \\ Department of Chemistry, Temple University, Philadelphia, PA 19122, USA
}

\begin{abstract}
The classical turning radius $R_{t}$ of an atom can be defined as the radius where the KS potential is equal to the negative ionisation potential of the atom, i.e. where $v_{s}\left(R_{t}\right)=\epsilon_{h}$. It was recently shown [P.N.A.S. 115, E11578 (2018)] to yield chemically relevant bonding distances, in line with known empirical values. In this work we show that extension of the concept to non-integer electron number yields additional information about atomic systems, and can be used to detect the difficulty of adding or subtracting electrons. Notably, it reflects the ease of bonding in open $p$-shells, and its greater difficulty in open $s$-shells. The latter manifests in significant discontinuities in the turning radius as the electron number changes the principal quantum number of the outermost electronic shell (e.g. going from $\mathrm{Na}$ to $\mathrm{Na}^{2+}$ ). We then show that a non-integer picture is required to correctly interpret bonding and dissociation in $\mathrm{H}_{2}^{+}$. Results are consistent when properties are calculated exactly, or via an appropriate approximation. They can be interpreted in the context of conceptual density functional theory.
\end{abstract}

\section{INTRODUCTION}

Density functional theory (DFT) is the dominant approach for doing chemistry on computers[1, 2]. Thousands of papers each year report studies of systems as diverse as biological molecules and technological materials. The number of such studies is increasing faster than the rate of chemistry and physics papers. Almost all of these use some form of density functional approximation, such as B3LYP[3] or PBE[4], which make quantum mechanical calculations tractable. The insights offered by DFT studies are increasingly appreciated by theoretical and experimental research teams.

What is less widely appreciated is that DFT is also an exact reformulation of the Schödinger equation, which is typically then approximated. Thus, auxilliary properties within DFT, such as the Kohn-Sham (KS) effective potential, might be expected to provide conceptual insights into the behaviour of electronic systems, just as properties of wave function theories do. Indeed, recent work by Ospadov et al[5] showed that the classical turning radius, where the energy of the highest occupied orbital equals the energy of the confining KS potential, provided a chemically intuitive bond length parameter, obtainable from first principles calculations of free atoms and molecules. In classical pysics, even the most energetic electron would be confined inside this surface.

Such a result falls within the context of conceptual

\footnotetext{
*Electronic address: t.gould@griffith.edu.au
}

DFT - in which density functionals are used to provide or justify traditional chemical concepts via mathematical rigour. It is with this goal in mind that we here extend the results of previous work by extending analysis to continuous (fractional) electron number. We thus provide understanding of what happens when electrons are continuously added or removed from atoms. Such a result offers insights into how bonding forms, in addition to the nature of such bonds.

\section{METHOD}

Let us briefly summarise DFT in the Kohn-Sham (KS) formalism. We first recognise that the groundstate of any electronic system described by the Schrödinger equation $\hat{H}=\hat{T}+\hat{W}+\int v(\mathbf{r}) \hat{\rho}(\mathbf{r}) d \mathbf{r}$ is entirely determined by its external (usually nuclear) potential $v$ which acts only on the number density operator $\hat{\rho}$. The kinetic energy term $\hat{T}=-\frac{1}{2} \sum_{i=1}^{M} \nabla_{i}^{2}$ and electron-electron interaction term $\hat{W}=\sum_{i>j}\left|\mathbf{r}_{i}-\mathbf{r}_{j}\right|^{-1}$ are universal to electrons. All terms are given in atomic units here and throughout, unless otherwise indicated.

Using these properties lets us write[6]:

$$
\begin{aligned}
& E_{0}^{M}[v]=\min _{\rho^{M}}\left\{F\left[\rho^{M}\right]+\int \rho^{M}(\mathbf{r}) v(\mathbf{r}) d \mathbf{r},\right. \\
& F\left[\rho^{M}\right]=\min _{|\Psi\rangle \rightarrow \rho^{M}}\langle\Psi|\hat{T}+\hat{W}| \Psi\rangle,
\end{aligned}
$$

for $M$ electrons in external potential $v$. Here, we have simply split the usual minimisation over wavefunctions into two parts: one over $M$-electron densities $\rho^{M}$ [in 
(1)] and one over Fermionic $M$-electron wavefunctions $|\Psi\rangle$ constrained to give the desired density [in (2)]. The potential $v$ may be reciprocally determined by the density.[1]

KS DFT[2] introduces an additional functional, $T_{s}\left[\rho^{M}\right]=\min _{|\Phi\rangle \rightarrow \rho^{M}}\langle\Phi|\hat{T}| \Phi\rangle$, which makes calculations tractable. Because the kinetic energy operator is a onepoint operator (i.e., it can be expanded as one-electron terms), the minimising $|\Phi\rangle$ takes the form of a Slater determinant with ordered (on $\epsilon_{i}$ ) orbitals that are solutions of

$$
\left[-\frac{1}{2} \nabla^{2}+v_{s}\left(\left[\rho^{M}\right], \mathbf{r}\right)\right] \phi_{i}(\mathbf{r})=\epsilon_{i} \phi_{i}(\mathbf{r})
$$

Here, $v_{s}\left[\rho^{M}\right]=\delta\left(F\left[\rho^{M}\right]-T_{s}\left[\rho^{M}\right]\right) / \delta \rho^{M}$, is the multiplicative $\mathrm{KS}$ potential, $\epsilon_{i}$ is a $\mathrm{KS}$ single-particle energy, and $\phi_{i}$ is a KS orbital. These quantities exclusively determine the properties of the KS representation of the interacting system.

Due to degeneracy in spin, the highest occupied molecular orbital (HOMO) that appears in the Slater determinant, $h$, is the smallest integer greater than or equal to half the number of electrons. If everything is carried out exactly, $\epsilon_{h}=E_{0}^{M}-E_{0}^{M-1}$ - i.e. it equals the negative ionisation potential (or electron affinity for anions). The density is

$$
\rho^{M}=\sum_{i} \theta_{i}\left|\phi_{i}\right|^{2} \equiv \sum_{i} \theta_{i} \rho_{i}
$$

where $\theta_{i}=2$ (for $\left.i<h\right), 1$ or 2 (for $i=h$-depending on whether the electron number is odd or even) and 0 otherwise. When $v_{s}$ is approximated, neither $\epsilon_{h}$ nor $\rho$ obtain their exact values. Equation (4) provides an alternative definition for $v_{s}[\rho]$ : it is the potential appearing in $(3)$ whose orbitals give the desired density $\rho$ via (4). Thus, given a density $\rho$ it is possible to determine the potential $v_{s}$ via a process known as density inversion. [7-11]

Ospadov et al[5] recently showed that the classical turning radius,

$$
R_{t}, \text { s.t. } v_{s}\left(R_{t}\right)=\epsilon_{h},
$$

takes on a unique value in atoms, which defines a chemically meaningful length that can indicate the character of bonds in molecules. Notably, the ratio $\beta(A B)=$ $E^{A B} /\left(R_{t}^{A}+R_{t}^{B}\right)$ of the bond-length $D_{A B}$ between atoms $A$ and $B$ to the sum of their turning radii $R_{t}^{A}$ and $R_{t}^{B}$, yielded values of $\approx 0.5$ for covalent bonds, $\approx 1.0$ for ionic bonds, and $\approx 1.5$ for weaker bonds.

Moreover, this success was obtained using an approximation to DFT - specifially by finding the KS potential corresponding to the atomic density found via HartreeFock theory. In some cases this was compared to results found via more (and less) accurate approximations, and shown to yield similar results in all cases. This apparent (and perhaps surprising) universality of the turning radius let the authors obtain $R_{t}$ turning radii for all atoms in the first five rows of the periodic table, including first and second cations for most elements, and some anions.

It might seem that the story of the remarkable success of $R_{t}$ as a first principles based indicator of chemical properties is almost complete - certainly one is unlikely to find too much of interest in third cations. However, as we shall proceed to show, by extending the concept to fractional ions by connecting the system to a distant bath, (i.e., systems with an integer nuclear charge $Z$, but real number of electrons $N$ ) even more chemistry can be revealed.[12]

To deal with fractional ions we turn to fractional ensemble DFT[13, 14] (EDFT) which can be viewed conceptually as connecting the system to a distant bath which is adjusted to maintain the desired fractional number of electrons (e.g., by taking a series of exactly degenerate copies of the system). In EDFT, expectation values of pure state wavefunctions $\left|\Psi^{M}\right\rangle$ in $F[\rho]$ and $T_{s}[\rho]$ are replaced by traces over ensemble density matrices $\hat{\Gamma}=(1-c)\left|\Psi^{M}\right\rangle\left\langle\Psi^{M}|+c| \Psi^{M+1}\right\rangle\left\langle\Psi^{M+1}\right|$ for the interacting state; and $\hat{\Gamma}_{s}=(1-c)\left|\Phi^{M}\right\rangle\left\langle\Phi^{M}|+c| \Phi^{M+1}\right\rangle\left\langle\Phi^{M+1}\right|$ for the non-interacting one. Here, $c=N-M>0$ is the fractional charge, and $M$ is the largest integer less than $N$. It follows that,

$$
\rho^{N}=(1-c) \rho^{M}+c \rho^{M+1}
$$

can be obtained from, e.g. $\rho=2 \sum_{i<h} \rho_{i}+c \rho_{h}$, for even $M$, or $\rho=2 \sum_{i<h} \rho_{i}+(1+c) \rho_{h}$ for odd $M$. In our ensembles, degenerate states are assigned equal weights, so that all atoms have spherically-symmetric densities and Kohn-Sham potentials. Consequently, the orbital expressions given above for $\rho$ can become more complicated in practice. (see, e.g., discussion in Sec 3 of ref. [15] for further details)

The ability to write $\rho^{N}$ in terms of orbitals allows us to obtain $v_{s}$ via density inversion, provided we know the density for $M$ and $M+1$ electron systems[11]. Thus, we can define a continuous turning radius via

$$
v_{s}^{N}\left(R_{t}^{N}\right)=\epsilon_{h}^{M+1},
$$

where we used the exact relationship $\epsilon_{h}^{N}=\epsilon_{h}^{M+1}$, for the HOMO orbital energy[14] at non-integer $N$, the so-called Koopmans' theorem for DFT.[16]

We shall now proceed to investigate $R_{t}^{N}$ for a selection of key atoms for which exact results can be obtained. We supplement these calculations with some additional, approximate calculations using $\rho^{N} \approx(1-c) \rho_{A}^{M}+c \rho_{A}^{M+1}$ to further elucidate results, where $\rho_{A}$ indicate densities obtained from an approximation at integer electron number. We then theoretically explain the revealed behaviour. Finally, we conclude.

Before continuing, however, we shall make note of an important known behaviour of the KS potential that affects interpretation throughout the next sections. The exact KS potential $v_{s}^{N}$ for $N$ infinitesimally less than the integer $M$, which we call $v_{s}^{M}$, tends to zero as $|\mathbf{r}| \rightarrow$ $\infty$. When $N$ crosses above $M$, the exact Kohn-Sham 
jumps up by a position-independent positive exchangecorrelation discontinuity $\Delta_{\mathrm{xc}}^{M}$. For most of the cases considered here,

$$
\Delta_{\mathrm{xc}}^{M}=I_{M}-A_{M},
$$

is the difference between the first ionization energy $I_{M}$ and the electron affinity $A_{M}$ of the real $M$-electron system. More generally (e.g., when $M$ closes a subshell, as at $M=2$ and 4$), \Delta_{\text {xc }}^{M}=\left(I_{M}-I_{M}^{\text {non }}\right)-\left(A_{M}-A_{M}^{\text {non }}\right)$ where "non" denotes a system of $M$ non-interacting electrons in external potential $v_{s}^{M}$. This discontinuity may be expected to manifest in the turning radius of atoms, as $N$ is varied continuously.

\section{RESULTS}

Let us begin by selecting some interesting atoms. Since we would like to control for sources of error, we select $\mathrm{Li}, \mathrm{C}$ and $\mathrm{F}$, for which we have high-quality numerically exact densities and energies, for the neutral atom, cation and anion. For each of these elements, Gould and Toulouse[11] parametrised densities from quantum Monte Carlo (QMC) calculations[17, 18], and then inverted them to obtain the exact $\rho^{N}, v_{s}^{N}$ and $\epsilon_{h}^{N}=\epsilon_{h}^{M+1}$ for $Z-1 \leq N \leq Z+1$. Additional exact data can be obtained analytically, by recognising that for $N=1$ any elemental system is Hydrogenic, giving, in atomic units, $v_{s}=-Z / r$ and $\epsilon_{h}=-Z^{2} / 2$. We are thus able to find $R_{t}^{N}$ for a continuous range of $N$ values, and for $N=1$, where $R_{t}^{N=1}=2 / Z$.

To demonstrate how the turning radius changes with electron number, Figure 1 show plots of $R_{t}^{N}$ for a range of $N$ values for the elements Li, C and F. Here $R_{t}^{N}$ is obtained exactly for a range of values (indicated by markers), and also evaluated using the following two fits:

Fit 1: Quadratic fit through $N \in\{1, Z-1, Z, Z+1\}$;

Fit 2: Quadratic fit through $N \in\{Z-1, Z, Z+1\}$.

Fit 1 minimizes the root-mean-square error for the four given integer values of $N$, while Fit 2 is exact at $N$ just below the three given integer values. Fit 2 was used in ref. [5] to predict the turning radius of metastable $\mathrm{O}^{2-}$ from those for $\mathrm{O}^{+}, \mathrm{O}$ and $\mathrm{O}^{-}$, and that prediction is well justified by the present work.

We note that the values we obtained using exact densities for turning radii at integer $N$ are very close to those found using exact exchange theory by Ospadov et al[5] - typically within a few picometers and at worst within $10 \mathrm{pm}$. This further validates the argument that turning radii are somewhat independent of approximations.

It is immediately clear that for $\mathrm{C}$ and $\mathrm{F}$, the turning radius changes pretty much continuously with perhaps a small derivative discontinuity as $N$ passes through integers. This small deviation becomes more apparent when comparing against Fit 2, which is the exact quadratic fit through the cation, atom and anion. For these cases, Fit
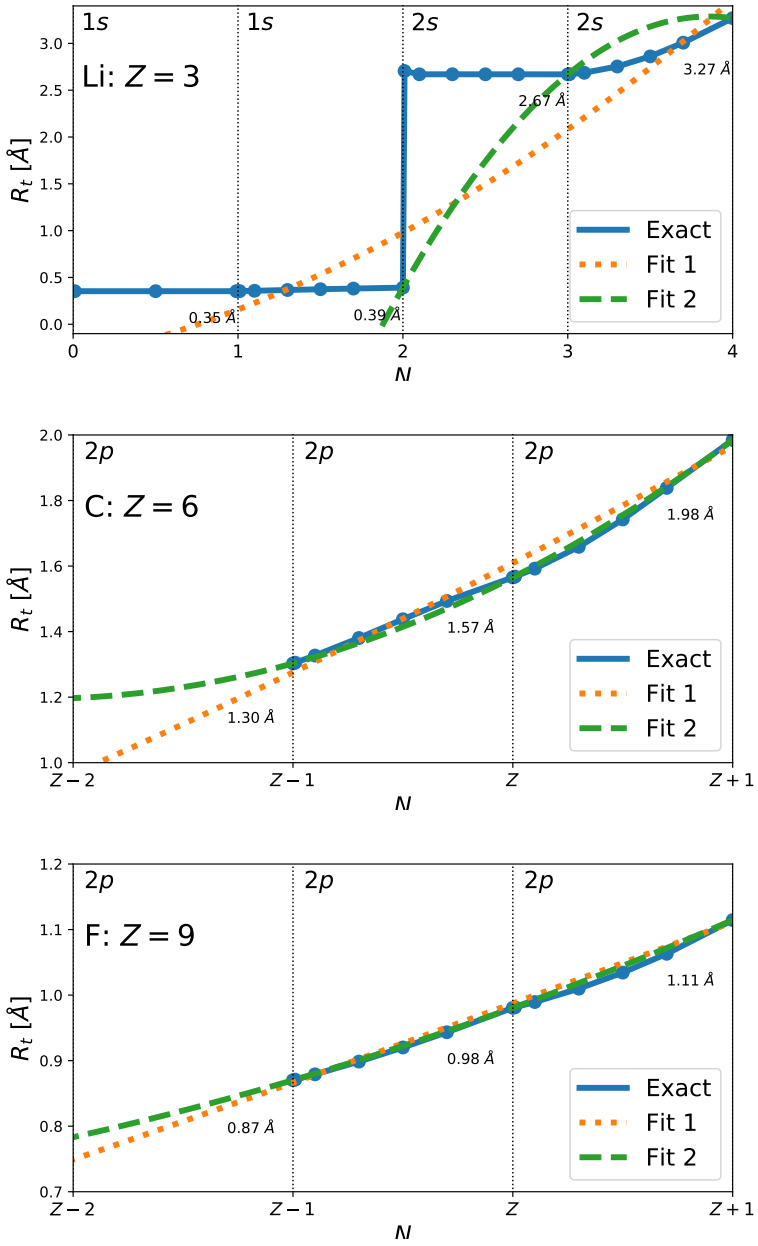

FIG. 1: Turning radius $R_{t}^{N}$ of Li (top), C (middle) and F (bottom) for continuous changes in $N$. The solid line shows the exact results, with markers indicating the values of $N$ used in calculations. The dashed and dotted lines show the two fits. Numbers provide the turning radius at integer values of $N$, in $\AA$. Vertical lines indicate integers, and shells are indicated at the top of the plot.

1, which adds $N=1$ to the quadratic fit is still pretty good for $Z-1 \leq N \leq Z+1$, showing how well-behaved the turning radius is.

For Li, however, we get dramatically different results. The turning radius changes smoothly for anions, as with $\mathrm{C}$ and $\mathrm{F}$. But for cations it remains essentially constant for continuous changes to $N$, and then changes abruptly at integers giving it a "stepped" structure.

To gain further insight into this effect, in Figure 2 we supplement our exact results for Li by the equivalent plot for $\mathrm{Na}$, but using exact exchange (EXX) data obtained at integer values for $\mathrm{Na}$ and its ions. These densities are then interpolated using (6) to obtain densities at noninteger $N$. Potentials $v_{s}^{N}$ are found via inversion. We expect EXX to be a very good approximation of the turning radius in this case, based on the success of EXX in 


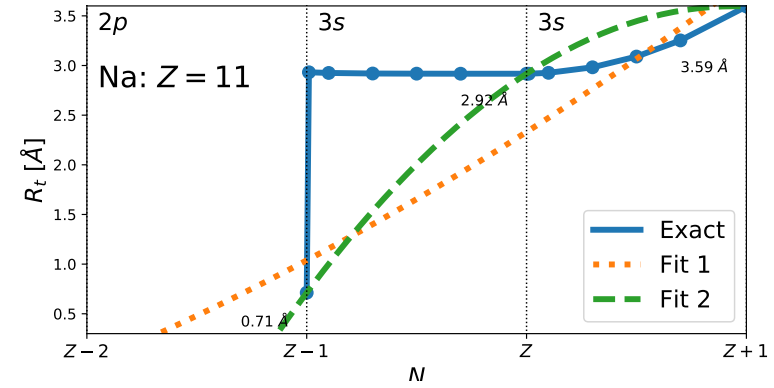

FIG. 2: Like Figure 1, but for Na using exact exchange densities.

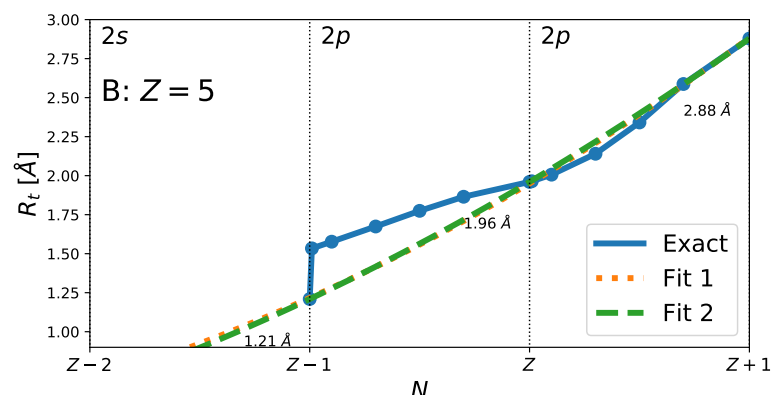

FIG. 3: Like Figure 1, but for B using exact exchange densities.

describing $s$-electrons, and because turning radii seem to be reasonably stable with respect to approximations.

We see that $\mathrm{Na}$ has exactly the same qualitative behaviour - smooth variation in $R_{t}^{N}$ for anions, but jumps for cations. This suggests that the turning radius indicates some sort of fundamental difference between these two cases. In the case of passing through a shell, this behaviour might be expected - the physics of $s$-electrons (e.g.) are fundamentally different to those of $p$-electrons. But in $\mathrm{Li}$ and $\mathrm{Na}$ we get the behaviour as the outermost $s$-electron is removed, indicating something interesting. In the next section we explain this result theoretically.

Finally, in Figure 3 we show results for B (using EXXderived densities), which also involves a change to the shell structure. Here, we get the same qualitative features for cations as for $\mathrm{Li}$ and $\mathrm{Na}$, but rather than a more-orless constant $R_{t}$ we find a slow decrease, followed by only a mild discontinuity as $N \rightarrow Z-1$. As we shall discuss in the penultimate section, this result is also consistent with theory and chemical intuition.

\section{A. Theory}

Let us now seek to understand the unusual stepwise behaviour of the turning radius. To begin, we can see that it is always present for $N<1$, regardless of the nuclear charge. For exactly one-electron $(N=1)$, we have a simple Hyrdogenic atom. Thus, by definition

$$
v_{s}^{N=1}=-\frac{Z}{r}=\frac{\nabla^{2} \sqrt{\rho^{N=1}}}{2 \sqrt{\rho^{N=1}}}-\epsilon_{h}^{N=1}, \quad \epsilon_{h}^{N=1}=-\frac{Z^{2}}{2}
$$

giving $R_{t}^{N=1}=2 / Z$. However, for $N<1$ atom we get a density $\rho^{N}=N \rho^{1}$, so that $v_{s}^{N<1}=v_{s}^{N=1}$. We can then combine this with $\epsilon_{h}^{N}=\epsilon_{h}^{M+1}=\epsilon_{h}^{N=1}$ to see that

$$
R_{t}^{N}=\frac{2}{Z}, \quad \forall 0<N \leq 1 .
$$

Thus, the turning radius does not vary for $N<1$. For $N=0$ it is difficult to define the turning radius at all.

Next, we extend our analysis to more complex cases. To begin, we use the finding from Gould and Toulouse[11] that,

$$
\begin{aligned}
\rho^{N} v_{s}^{N} \approx & (M+1-N) \rho^{M}\left(v_{s}^{M}+\Delta_{\mathrm{xc}}^{M}\right) \\
& +(N-M) \rho^{M+1} v_{s}^{M+1}
\end{aligned}
$$

is a remarkably good approximation for the KS potential at non-integer electron number $N \neq M$, especially for $N-M$ not too large. Here, $\Delta_{\mathrm{xc}}^{M}$ [see Eq. (8)] is the derivative discontinuity of the energy $E^{N}$, which introduces a step change in $v_{s}^{N}$ as it passes through an integer, i.e. $\lim _{\eta \rightarrow 0^{+}} v_{s}^{M+\eta}-v_{s}^{M-\eta}=\Delta_{\mathrm{xc}}^{M}$, a behaviour captured by the approximation [see (8)]. Thus, we have an analytic approximation for $v_{s}^{N}$ at non-integers, in terms of their properties at integers.

For $c=N-M$ small but finite, we find

$$
v_{s}^{N}(r)-v_{s}^{M}(r) \approx \Delta_{\mathrm{xc}}^{M} S^{N}(r) .
$$

We can use the right Fukui function $f_{+}^{M}=\rho^{M+1}-\rho^{M}$ to re-express

$$
S^{N}(r)=\frac{\rho^{M}(r)}{\rho^{N}(r)}=\frac{\rho^{M}(r)}{\rho^{M}(r)+c f_{+}^{M}(r)} .
$$

$S^{N}(\mathbf{r})$ is the ratio of the $N=M$ density to the fractional electron density, which for any finite $c$ must be 1 for sufficiently small $r$ and 0 for sufficently large $r$, because $\rho_{M+1}$ decays with $r$ more slowly than $\rho_{M}$.

There is, however, an order of limits issue here, due to the competing scale of $c f_{+}^{M}(r)$ and $\rho^{M}(r)$ for any given finite $r$. For very small $r$, the density $\rho^{M} \gg c f_{+}^{M}$, and $S^{N}(r)=1$. Similarly, for $r$ sufficiently large and $c$ finite we find $c f_{+}^{M}>\rho^{M}$, and $S^{N}(r)=0$. These are the cases mentioned above. However, for some mid-sized values of $r$, the two densities in the denominator of Eq. (13) obey $\rho^{M} \rightarrow C \exp \left(-k^{M} r\right)$, and $f_{+}^{M} \rightarrow C^{\prime} \exp \left(-k^{M+1} r\right)$ (which decays slower) - for $k^{N}=2\left[-2 \epsilon_{h}^{N}\right]^{\frac{1}{2}}$. Thus, there exists a

$$
R_{c}(c)=\frac{-\ln c+B}{k^{M}-k^{M+1}}
$$

where $B$ is constant, such that for $0<c \ll 1$ and $r \approx$ $R_{c}(c)$, the two densities in the denominator of (13) are approximately equal, giving $S^{N}\left(R_{c}(c)\right) \approx \frac{1}{2}$. 

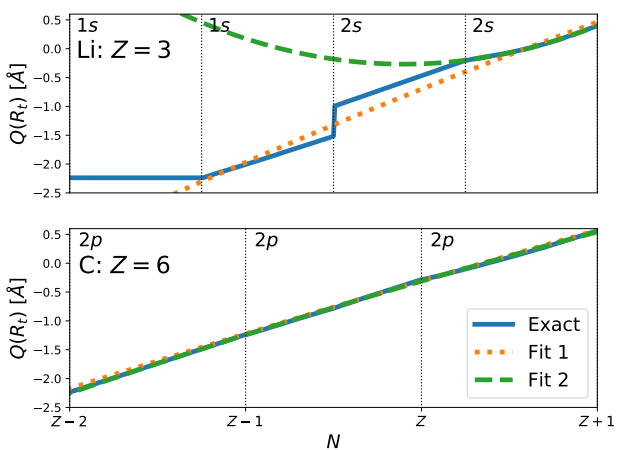

FIG. 4: Effective charge $Q\left(R_{t}\right)=R_{t} v_{s}\left(R_{t}\right)$ at the turning radius for ions of $\mathrm{Li}$ and $\mathrm{C}$. Fits (as previous) are included to illustrate approximate continuity, or otherwise.

Now let us consider the turning radius $R_{t}^{M}$, where $v_{s}^{M}\left(R_{t}^{M}\right)=\epsilon_{h}^{M}$. For small $c$, we find from Eq. (12),

$$
v_{s}^{N}\left(R_{t}^{M}\right) \approx \epsilon_{h}^{M}+\Delta_{\mathrm{xc}}^{M} S^{N}\left(R_{t}^{M}\right) .
$$

We must consider two cases. In one case, (e.g., when subshells don't change) we find $R_{t}^{M} \ll R_{c}(c)$ so that $S^{N}\left(R_{t}^{M}\right) \approx 1$ and

$$
v_{s}^{N}\left(R_{t}^{M}\right) \approx \epsilon_{h}^{M}+\left(\epsilon_{h}^{M+1}-\epsilon_{h}^{M}\right)=\epsilon_{h}^{N},
$$

because $\epsilon_{h}^{N}=\epsilon_{h}^{M+1}$. Thus $R_{t}^{N} \approx R_{t}^{M}$ and the turning radius changes only slowly with increasing electron number.

By contrast, the other case gives $R_{t}^{M} \gg R_{c}(c)$ so $S^{N}\left(R_{t}^{M}\right) \approx 0$ and we instead find

$$
v_{s}^{N}\left(R_{t}^{M}\right) \approx \epsilon_{h_{M}}^{M}<\epsilon_{h_{N}}^{N} \equiv \epsilon_{h_{M+1}}^{M+1} .
$$

Here, we used the more complex version of Eq. (8), given in the text below the equation, expanded in terms of KS eigenvalues (because of Koopman's theorem) $-h_{M}$ indicates the HOMO for $M$ electrons. Since $v_{s}^{N}$ behaves approximately as $-Q^{M+1} / r\left(\right.$ where $\left.Q^{M+1}=M-Z\right)$ at the turning radius, we get

$$
R_{t}^{N} \approx \frac{Q^{M+1}}{\Delta_{\mathrm{xc}}^{M}} \gg R_{t}^{M}=\frac{Q^{M}}{\epsilon_{h}^{M}} .
$$

In this case, there is an extremely rapid change of the turning radius. In fact, as can be seen in Figure 4, which shows $Q^{N}\left(R_{t}^{N}\right)=R_{t}^{N} v_{s}^{N}\left(R_{t}^{N}\right)$ for Li and C, this approximation is only crudely correct. Nonetheless, we see that $Q^{N}\left(R_{t}^{N}\right)$ varies smoothly and continuously for C, but abruptly for $\mathrm{Li}$, validating the core of the argument.

Thus, we can finally explain what happens when the first electron is filled in an $S$-shell. In such a case, $\left|\epsilon_{h}^{M}\right|=$ $o(1) \mathrm{Ha}$ and $\left|\epsilon_{h}^{M+1}\right|=o(0.1) \mathrm{Ha}$, since the cation of an open $S$-shell atom is always much more tightly bound than the neutral species. Thus, (14) has a small prefactor
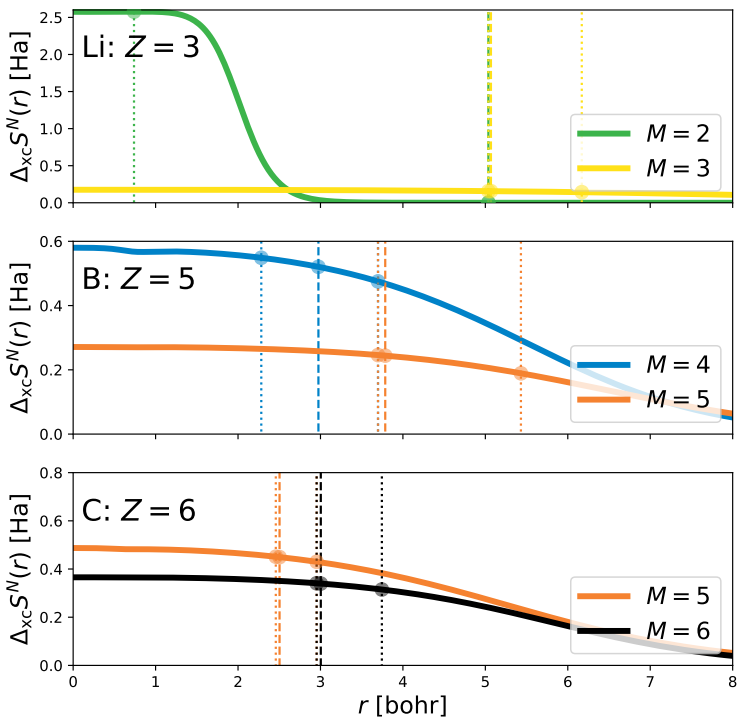

FIG. 5: $\Delta_{\mathrm{xc}}^{M} S^{N}(r)$ versus $r$ for atoms and cations of $\mathrm{Li}$ and C, for $M \in\{Z-1, Z\}$ and with $N=M+0.1$. Vertical lines indicate turning radii at integers $M \in\{Z-1, Z, Z+1\}$ (dotted) and and for integers plus small constants $N \in\{Z-$ $0.9, Z+0.1\}$ (dashed).

and is small for even quite small values of $c$. This means that $R_{t}^{M} \gg R_{c}$ and thus $R_{t}^{N} \gg R_{t}^{M}$ varies rapidly. In the other cases, where $\epsilon_{h}^{M} \approx \epsilon_{h}^{M+1}$, we instead find a small denominator in the expression for $\ln (c)$, meaning that $R_{c} \gg R_{t}^{M}$ and $R_{t}^{N} \approx R_{t}^{M}$.

We finally illustrate this argument visually by showing in Figure 5 the values of $S^{N}(r)$ for atoms and cations of $\mathrm{Li}, \mathrm{B}$ and $\mathrm{C}$. For $\mathrm{C}$, we see that $\Delta_{\mathrm{xc}}^{M} S^{N}(r)$ varies smoothly at the values of the turning radii. Furthermore, the curves for the atom and cation are similar, meaning that $R_{t}$ varies smoothly. By contrast, for $\mathrm{Li}$ the step is large, rapid, and found in between $R_{t}^{N=Z}$ and $R_{t}^{N=Z-1}$. Also, the atom and cation have dramatically different behaviours. Thus, $R_{t}$ remains basically constant for $2<N \leq 3$ and changes abruptly at $N=2$. For $\mathrm{B}$, the difference is somewhere in between, with smooth curves but a significant difference between the atom and cation. Keeping in mind the simplicity of our model, this explains why its turning radius $R_{t}$ has a small step, in combination with a smooth transition.

\section{DISCUSSION}

\section{A. Atoms}

We have so far shown how the classical turning radius varies with electron number $N$ for "normal" cases $\mathrm{C}$ and $\mathrm{F}$ and anions of $\mathrm{Li}$ and $\mathrm{Na}$, where $R_{t}$ varies smoothly in 


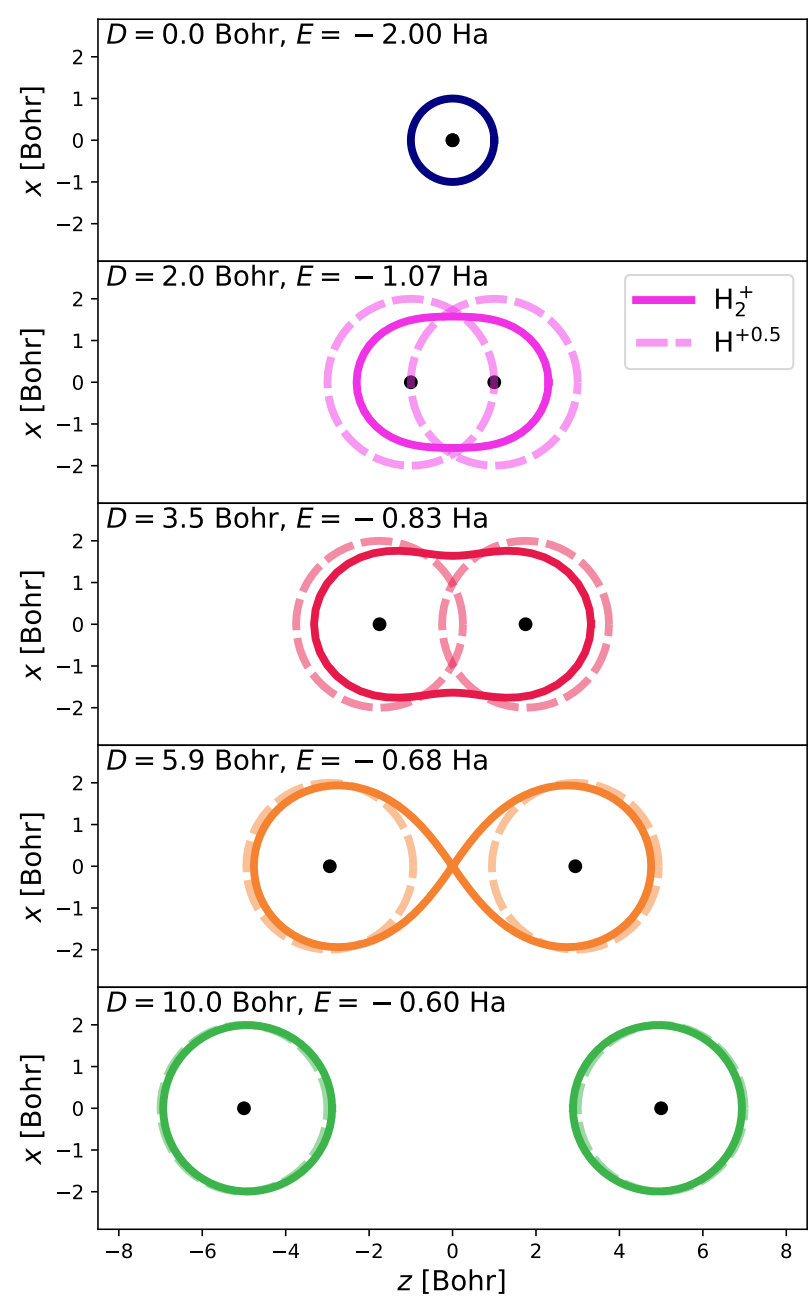

FIG. 6: Classical turning surface for $\mathrm{H}_{2}^{+}$(two protons at separation $D$, and one electron), shown at different values of $D$ along the dissociation curve from the unified-atom limit $(D=0)$ to the dissociation limit $(D \rightarrow \infty)$. The intersection of this surface with the $x z$-plane is plotted here. The dashed lines show the classical turning radius of both $\mathrm{H}$ atoms, which are equal to those of $\mathrm{H}^{+\frac{1}{2}}$ per present work. Note that the bond "breaks" at $D=5.9 \mathrm{Bohr}$, in the range of equilibrium van der Waals bond lengths between atoms.

the range shown; and "stepped" cases like cations of $\mathrm{Li}$ and $\mathrm{Na}$, where the turning radius is more-or-less constant upon removal of the electron, until it changes suddenly; and $\mathrm{B}$, which has elements of both behaviours. We now turn to explaining the meaning of these findings.

The first thing to consider is what it doesn't represent. In all four of these cases we do not change the nodal structure of the orbitals. Thus, the sudden change cannot be explained by changes thereto. This might be an obvious first guess, as another interpretation of $R_{t}$ is the value at which $\nabla^{2} \phi_{h}=0[5]$, which one might expect to be related to the nodes.

Mathematically, we have seen that the sudden change corresponds to a large change to the HOMO energy, in combination with a rapdily varying $S^{N}(r)$ [eq. (13)]. Physically, this corresponds to a case where the HOMO and second-highest occupied molecular orbitals are very different - which happens when the orbital passes through a level (from $2 s$ to $1 s$ in the case of $\mathrm{Li}$ ).

Chemically, we observe that this is a somewhat intuitive result, albeit one that requires thinking about the implict properties of fractional electrons, rather than their explicit properties - that is, the properties they would like to have. $\mathrm{Li}$ and $\mathrm{Na}$ atoms wish to donate their outermost electron in full to form ionic bonds. Thus, there is a "pressure" from the bath to behave like the neutral atom, which continues until the fractonal electron is almost fully removed and the system become fully cationlike. This is reflected in the spontaneous step change in the turning radius as $N \rightarrow(Z-1)^{+}$. This result is perhaps more intuitive in the one-electron case, where any portion of an electron surrounding the $\mathrm{H}$-like nucleus acts exactly like a full electron.

The result for B (Figure 3) is also understandable by the same arguments. As with $\mathrm{Li}$ and $\mathrm{Na}$, the same step behaviour occurs in the atom-cation transition. But, due to the smaller gap between the $2 p$ and $2 s$ shells, its impact on the turning radius is smaller. Thus, we see an incomplete decrease in the turning radius, followed by a small step. The balance between the competing needs of the bath and fractional ion is consequently small, but finite.

By contrast, $\mathrm{C}$ and $\mathrm{F}$ are more flexible in their bonding, and can attract or gift electrons as part of a covalent bond. Thus their turning radii change only slowly to reflect small changes to electron number, due to a smooth interaction with the bath. Although chemically infeasible, this same behaviour is reflected in the anions of $\mathrm{Li}$, which must first fully lose the second electron before feeling the unusual effects on the cations.

A related perspective is that the unusual "stepped" features reflect strong correlations with the bath, caused by unbalanced energetics between the effective electrostatic (KS) potential $v_{s}$ at the turning radius, and the fictitious energy of the HOMO that must maintain the correct asymptotic profile of the density.

\section{B. Dissociation of $\mathbf{H}_{2}^{+}$diatom}

We complete our discussion by putting our results in a more chemical context. We thus consider a case where atoms attain a fractional charge as part of a dissociation process. This allows us to explore the behaviour of the classical turning surface for systems with non-integer components; and contextualise theory within the bondtype analysis of Ref. [5].

We study the diatomic $\mathrm{H}_{2}^{+}$cation as all relevant quantities can be obtained exactly. Its KS potential $v_{s}(\mathbf{r})=$ $-\frac{1}{|\mathbf{r}+D \hat{\mathbf{z}} / 2|}-\frac{1}{|\mathbf{r}-D \hat{\mathbf{z}} / 2|}$ at inter-atomic distance $D$ is equal to its external potential, and is thus trivial to obtain. 
Ground state electronic energies, $E(D)$ (not including the potential energy of Coulomb repulsion between the two protons), can be obtained exactly as a function of $D$, and it follows from Koopman's theorem that $\epsilon_{h}=E(D)$. Thus, the exact classical turning surface is obtained by finding values, $\mathbf{R}_{t}$, obeying

$$
-\frac{1}{\left|\mathbf{R}_{t}+\frac{D \hat{\mathbf{z}}}{2}\right|}-\frac{1}{\left|\mathbf{R}_{t}-\frac{D \hat{\mathbf{z}}}{2}\right|}=E(D) .
$$

The turning surface is shown in Figure 6 for $\mathrm{H}_{2}^{+}$, with $D$ set to key values along the dissociation curve. We show only the $x-z$ plane, due to the cylindrical symmetry. The classical turning surface is obtained by solving Eq. (19) numerically. When $D=0$, the system is equivalent to one-electron $\mathrm{He}^{+}$, and thus has an analytic turning radius of $R_{t}(D=0)=2 / Z=1$ Bohr [Eq. (10)]. For $D>0$, we also show the classical turning radius of $\mathrm{H}\left(R_{t}=2 \mathrm{Bohr}=\right.$ $1.06 \AA)$ in our plots. By the arguments presented in this work, this should be the same as the turning radius of $\mathrm{H}^{+\frac{1}{2}}$.

It is clear from the figure that results for $\mathrm{H}_{2}^{+}$are consistent with the arguments laid out here, and inconsistent with earlier work[5]. The system dissociates $\left(D=10\right.$ Bohr) to two free $\mathrm{H}^{+\frac{1}{2}}$ atoms with $R_{t}=2 \mathrm{Bohr}$ $(1.06 \AA)$, per eq. (10). The equilibrium bond length of $\mathrm{H}_{2}^{+}$is $D=2$ Bohr $(1.06 \AA)$, which is shown in the second row of the figure. It is clear both theoretically and from the figure that the bond-length is half the sum of the classical turning radii. This gives a bond-type ratio

$$
\beta(A B)=\frac{D_{A B}}{R_{t}^{A}+R_{t}^{B}}=\frac{2}{2+2}=\frac{1}{2},
$$

(where $A$ and $B$ are $\mathrm{H}^{+\frac{1}{2}}$ ) consistent with the description of covalent bonding given by Ospadov et al[5]. This chemically intuitive result further supports the case for considering fractional classical turning radii.

Finally, we note that another interpretation of $\mathrm{H}_{2}^{+}$dissociation is that the molecule splits into a superposition of states with the electron localised on either atom. In this interpretation, the correct way to calculate the bondtype ratio is to set $A$ as $\mathrm{H}^{+}$and $B$ as $\mathrm{H}$ (or vice versa) in Eq. (20). Under this interpretation we must define the classical turning radius of $\mathrm{H}^{+}$to be the same as for $\mathrm{H}$ (2 Bohr), which is consistent with the limit $q \rightarrow 1^{-}$ $\left(N \rightarrow 0^{+}\right)$in $\mathrm{H}^{+q}$.

Figure 3 of Ref. 5 presented an intuitive but nonrigorous radius of $0 \mathrm{Bohr}$ for $\mathrm{H}^{+}$. That would make the bonding ratio (of $\left.\mathrm{H}_{2}^{+}\right) \beta(A B)=2 /(0+2)=1$, which is more typical of ionic bonding than the covalent bonding that is known to be present in $\mathrm{H}_{2}^{+}$.
The molecule $\mathrm{HeH}^{+}$is similar to $\mathrm{H}_{2}^{+}$and has $\beta_{A B}=$ $1.47 /(1.17+2)=0.46$, again in the covalent range of this ratio.

\section{CONCLUSIONS}

In this manuscript we generalized the recently introduced concept of the "classical turning radius" [5] of the KS potential for an atom, to account for non-integer electron number. We were thus able to explore how the turning radius varied with coninuous variations in electron number.

Our discussion at the beginning of Section III A leads to a correction to Fig. 3 of ref. [5]. That figure assumeed that the classical turning radius of $\mathrm{H}^{+}$is 0 , but (in the limit of infinitesimal positive electron number) it is actually 2 bohr $=1.06 \AA$, the same as for $H$.

Through analysis of numerically exact KS solutions of $\mathrm{Li}, \mathrm{C}$ and $\mathrm{F}$, and good exact exchange approximations for $\mathrm{Na}$ and $\mathrm{B}$, we showed that cations of $\mathrm{Li}$ and $\mathrm{Na}$ (and to a lesser extent B) have fundamentally different "stepped" behaviours, compared to the smooth behaviours of other species in our tests. We argued in Section IV A that this reflects the sudden and strong change of radius that occurs when the principal quantum number of the outermost and partly-occupied orbital changes suddenly, and the tendency of the $\mathrm{Li}$ and $\mathrm{Na}$ atoms to give up an electron to form a closed-shell positive ion.

The case of dissociating $\mathrm{H}_{2}^{+}$studied in Section IV B further confirms our approach. In our work, the fractional classical turning radius of $\mathrm{H}^{+\frac{1}{2}}$ is predicted to be the same as that of neutral $\mathrm{H}$. This result is consistent with well-dissociated $\mathrm{H}_{2}^{+}$. Furthermore, the bond length of $\mathrm{H}_{2}^{+}$gives a bond-type ratio indicating covalent bonding between $\mathrm{H}^{+\frac{1}{2}}$ atoms as defined here, according to the arguments (equations [7-9]) of Ospadov et al [5]. This result is consistent with chemical intuition, in which the electron is shared by both nucleii.

The results found here suggest that analysis of other conceptual properties of KS systems should be extended to continuous variation of electron number, to shed light on chemical bonding and other processes.

\section{Acknowledgments}

The work of JPP was supported by the US National Science Foundation (CMMT-Division of Materials Research, with a contribution from CTMC - Division of Chemistry) under Grant No. DMR-1607868. J.P.P. acknowledges discussions with Viktor N. Staroverov.
[1] P. Hohenberg and W. Kohn, Phys. Rev. 136, B864 (1964).

[2] W. Kohn and L. J. Sham, Phys. Rev. 140, A1133 (1965).
[3] A. D. Becke, J. Chem. Phys. 98, 1372 (1993).

[4] J. P. Perdew, K. Burke, , and M. Ernzerhof, Phys. Rev. Lett. 77, 3865 (1996). 
[5] E. Ospadov, J. Tao, V. N. Staroverov, and J. P. Perdew, Proceedings of the National Academy of Sciences 115, E11578 (2018).

[6] M. Levy, P.N.A.S. 76, 6062 (1979).

[7] Y. Wang and R. G. Parr, Phys. Rev. A 47, R1591 (1993).

[8] W. Yang and Q. Wu, Phys. Rev. Lett. 89, 143002 (2002).

[9] I. G. Ryabinkin and V. N. Staroverov, J. Chem. Phys. 137, 164113 (pages 8) (2012), URL http://link.aip. org/link/? JCP/137/164113/1.

[10] A. A. Kananenka, S. V. Kohut, A. P. Gaiduk, I. G. Ryabinkin, and V. N. Staroverov, J. Chem. Phys. 139, 074112 (pages 7) (2013), URL http://link.aip.org/ link/? JCP/139/074112/1.

[11] T. Gould and J. Toulouse, Phys. Rev. A 90, 050502 (2014).
[12] P. W. Ayers, J. Math. Chem. 43, 285 (2008).

[13] S. M. Valone, J. Chem. Phys. 73, 4653 (1980).

[14] J. P. Perdew, R. G. Parr, M. Levy, and J. L. Balduz, Phys. Rev. Lett. 49, 1691 (1982).

[15] T. Gould, S. Pittalis, J. Toulouse, E. Kraisler, and L. Kronik (2019).

[16] T. Tsuneda, J.-W. Song, S. Suzuki, and K. Hirao, J. Chem. Phys. 133, 174101 (pages 9) (2010), URL http: //link.aip.org/link/?JCP/133/174101/1.

[17] J. Toulouse, R. Assaraf, and C. J. Umrigar, J. Chem. Phys. 126, 244112 (2007).

[18] J. Toulouse and C. J. Umrigar, J. Chem. Phys. 128, 174101 (2008). 